\title{
Seropositivity in Myasthenia Gravis as a Predictor of Response to Therapeutic Plasma Exchange
}

\author{
Aktham Ismail Alemam ${ }^{\mathrm{a}, \mathrm{c}}$, Amal Ismail Abdulrahman ${ }^{\mathrm{b}}$
}

\begin{abstract}
Background: In spite of the increasing availability of immunomodulatory treatments for myasthenia gravis (MG), little is known about factors that predict response to the treatment. We aim to study if the presence of acetylcholine receptor (AChR) antibodies may be one of the predictors of the response to the therapeutic plasma exchange (TPE) in patients with MG.
\end{abstract}

Methods: The study was carried out in 78 patients with moderate to severe MG. They were divided into two groups: TPE group (62 patients) that included the patients who received TPE, and control (non-TPE) group who did not receive TPE and was only on medical treatment (16 patients). Patients in TPE group, then, were subdivided into sero-positive and sero-negative subgroups according to the presence or absence of AChR antibodies respectively. Scoring by Quantitative Myasthenia Gravis Scale (QMGS) was done before and 1 month after the TPE.

Results: After 1 month of treatment, the change in QMGS was significantly higher in the TPE group than in the Non-TPE one $(t=-6.406)(P<0.0001)$. In the sero-positive group, the score of QMGS ranged initially from 19 to 34 (mean $26.48 \pm 3.75$ ) and after 1 month from 11 to 30 (mean $18.00 \pm 4.45$ ) with the median change of -32. The QMGS change was significantly greater in the sero-positive group than in the sero-negative one $(\mathrm{t}=-3.516)(\mathrm{P}<$ $0.0001)$.

Conclusions: As regards to the presence of AChR antibodies in patients with MG, both sero-positive and sero-negative groups responded to TPE but the sero-positive group had a better response.

Keywords: Myasthenia gravis; Acetylcholine receptors; Seropositivity; Therapeutic plasma exchange

Manuscript submitted November 12, 2018, accepted December 12, 2018

aDepartment of Neuropsychiatry, Faculty of Medicine, Menoufia University, Shebin Elkom, Egypt

bDepartment of Anesthesiology, Faculty of Medicine, Menoufia University, Shebin Elkom, Egypt

${ }^{\mathrm{c} C}$ Corresponding Author: Aktham Ismail Alemam, Department of Neuropsychiatry, Menoufia University, Shebin Elkom, Egypt.

Email: e_aktham@yahoo.com

doi: https://doi.org/10.14740/jnr510
Introduction

Myasthenia gravis (MG) is a humoral auto-immune disease characterized by the presence of antibodies that are directed against the postsynaptic nicotinic acetylcholine receptors (AChRs) [1]. The incidence of the disease is from 0.25 to 2.0 per 1,000,000 and about $15-20 \%$ of the patients may suffer from myasthenic crisis with a fatality in up to $8 \%$ of them in this condition [2]. These antibodies are not present in normal people and considered highly specific to the MG. The highest levels (in up to $85 \%$ of cases) are present if $\mathrm{MG}$ is generalized, of early onset type, or associated with the presence of thymoma, while they are less frequently detected if $M G$ is mild or restricted as in ocular type [3]. The AChRs may be blocked by these antibodies leading to a decrease in the number of the available receptors in the membrane. The activation of the complement cascade may cause destruction of the endplate architecture and expansion of the synaptic cleft, increasing the distance for acetylcholine molecules to travel from their sites of release to their receptors [4]. Some studies have proved that the clinical improvement in response to immunomodulation was correlated with the reductions in AChR antibody levels.

Therapeutic plasma exchange (TPE) is one of the options of treatment of MG and it is recommended by the American Society for Pharesis as category I. TPE helps to remove the AChR antibodies, thus improving the neuromuscular junction transmission and increasing the muscle power [5]. Although there is a greater accessibility to immunomodulatory treatments, little data is available for the predictors of response to treatment [6]. This may put some obstacles in choosing the most effective and economic treatment for individual cases [7].

The aim of this study was to study if the presence of AChR antibodies may be one of the predictors of the response to the TPE in patients with moderate to severe generalized MG.

\section{Patients and Methods}

Between January 2016 and December 2017, 78 patients with MG completed the study. They were consecutively recruited from Neurology and Intensive Care Departments in Menoufia University Hospitals. The study was approved by the committee of ethics of Faculty of Medicine, Menoufia University.

The clinical severity was assessed according to Myasthenia Gravis Foundation of America (MGFA) classification, and the diagnosis was supported by electromyographic changes. 
MG was diagnosed in patients fulfilling at least two of the three criteria: 1) Presence of AChR antibodies; 2) Positive response to anticholinesterases; and 3) Abnormal neurophysiological findings (repetitive nerve stimulation and single-fiber electromyography).

Exclusion criteria included the myasthenia-like syndromes induced by drugs, such as D-penicillamine, antibiotics (e.g. ciprofloxacin), beta-adrenergic receptor blocking agents (e.g. propranolol), and cardiac drugs (e.g. verapamil). Also the exclusion involved patients who are contraindicated to plasmapheresis, such as those with the presence of hemorrhage or surgical bleeding, tumors, acute infectious processes as phlebitis, extreme degree of heart failure, and significant hypotension.

All the patients were diagnosed with moderate to severe MG. They were divided into two groups: TPE group that included the patients who received TPE sets and the non-TPE (control) group that included the patients who did not receive TPE sets and were on medical treatment only. Then the patients in TPE group were subdivided into sero-positive and sero-negative groups according to the presence or absence of AChR antibodies respectively.

The consent for the TPE was taken from the patient/ patient's relatives before the procedure. The patient's blood counts, electrolytes, serum proteins, coagulation profile, and vitals were checked, and abnormal parameters were corrected. The TPE was performed using a single volume plasma exchange with intermittent cell separator (Hemonetics MCS plus, kit 980/790) machines by femoral or central line access using 12 French double-lumen dialysis catheters. Five sessions were scheduled as one session every other day for 10 days. Anticoagulation with citrate was systematically used. The replacement of the plasma that was removed during the session was done with isotonic sterile saline, to make up onehalf of the volume and with $4 \%$ purified human albumin and fresh frozen plasma to complete it. A careful monitoring of hemodynamic parameters was done and complications during or following TPE were rapidly recognized and reverted by rationale interventions of medical staff that assisted the procedure.

The patients were subjected, initially and then 1 month after treatment, to scoring by the Quantitative Myasthenia Gravis Scale (QMGS). This is a validated clinical measure of MG disease severity developed by the MGFA, and is considered a clinical gold standard recommended for all prospective studies in MG. The scale measures ocular, bulbar, respiratory, and limb function, grades each finding, and ranges from 0 (no myasthenic finding) to 39 (maximum myasthenic deficits). The types of MG according to QMS were: mild $<11$ points, moderate 11 - 16 points, and severe $>16$ points. The score was calculated as the cumulative score of all the muscles [8].

\section{Statistical analysis}

Data were collected, and analyzed by the Statistical Package for the Social Science (IBM SPSS) version 23. The quantitative data were presented as mean, standard deviations and ranges when their distribution was found to be parametric, and as median with inter-quartile range (IQR) when their distribution was found to be non-parametric, while qualitative data were presented as number and percentages.

The comparison between two independent groups with qualitative data was done by using Chi-square test.

The comparison between two independent groups with quantitative data and parametric distribution was done by using independent $t$-test while the comparison for those with non-parametric data was done by using Mann-Whitney test.

\section{Results}

The TPE group included 62 patients with 46 women $(74.2 \%)$ and 16 men $(25.8 \%)$, with their age ranging from 18 - 60 years (mean $35.59 \pm 12.26)$ and duration of illness ranging from 12 84 months (mean $36.39 \pm 20.16$ ), and the control group included 16 patients with 11 women $(68.8 \%)$ and five men $(31.2 \%)$, with their age ranging from 18 - 60 years (mean $34.29 \pm 11.85$ ) and duration of illness ranging from 14 - 72 months (mean $42.47 \pm 18.19)$. According to the detection of AChR antibodies, the TPE group was subdivided into sero-positive group including $51(82.3 \%)$ patients and sero-negative group including $11(17.7 \%)$ patients.

The effects of TPE treatment on each individual parameter in QMGS of the patients were described in details in Table 1.

The QMGS score in the non-TPE group ranged initially from 21 to 27 (mean $24.14 \pm 2.00$ ), and from 22 to 32 (mean $26.37 \pm 3.20$ ) after 1 month, with a median change of 8 . The QMGS score in TPE group ranged initially from 18 to 34 (mean $25.66 \pm 3.85$ ), and from 11 to 30 (mean $18.05 \pm 4.14$ ) with median change of - 26.68. The change in QMGS was significantly higher in the TPE group than in the non-TPE one $(\mathrm{t}$ $=-6.406)(\mathrm{P}<0.0001)$ (Table 2).

In the sero-negative group, the QMGS score ranged initially from 18 to 27 (mean $22.33 \pm 3.03$ ) and after 1 month from 15 to 22 (mean18.27 \pm 2.43 ) with the median change of -14.88. In the sero-positive group, the QMGS score ranged initially from 19 to 34 (mean $26.48 \pm 3.75$ ) and after 1 month from 11 to 30 (mean $18.00 \pm 4.45$ ) with the median change of -32 . The change in QMGS was significantly greater in the sero-positive group than in the sero-negative one $(\mathrm{t}=-3.516)$ $(\mathrm{P}<0.0001)$ (Table 3).

\section{Discussion}

MG is an auto-immune disease caused by the failure of neuromuscular transmission, due to binding of autoantibodies to proteins involved in signaling at the neuromuscular junction [9]. Although there may be no consistent correlation between anti-AChR antibody serum titers and MG disease severity, patients with generalized MG tend to have higher antibody titers in comparison to the patients with ocular MG, and in individual patients, serial antibody titers tend to correlate with disease status [7]. Also, Romi et al found a correlation between serum AChR antibody titers and severity of MG [10]. Liu et al reported that although the clinical features of $\mathrm{MG}$ 
Table 1. Comparison for Each Parameter in the QMGS Before and After TPE Treatment

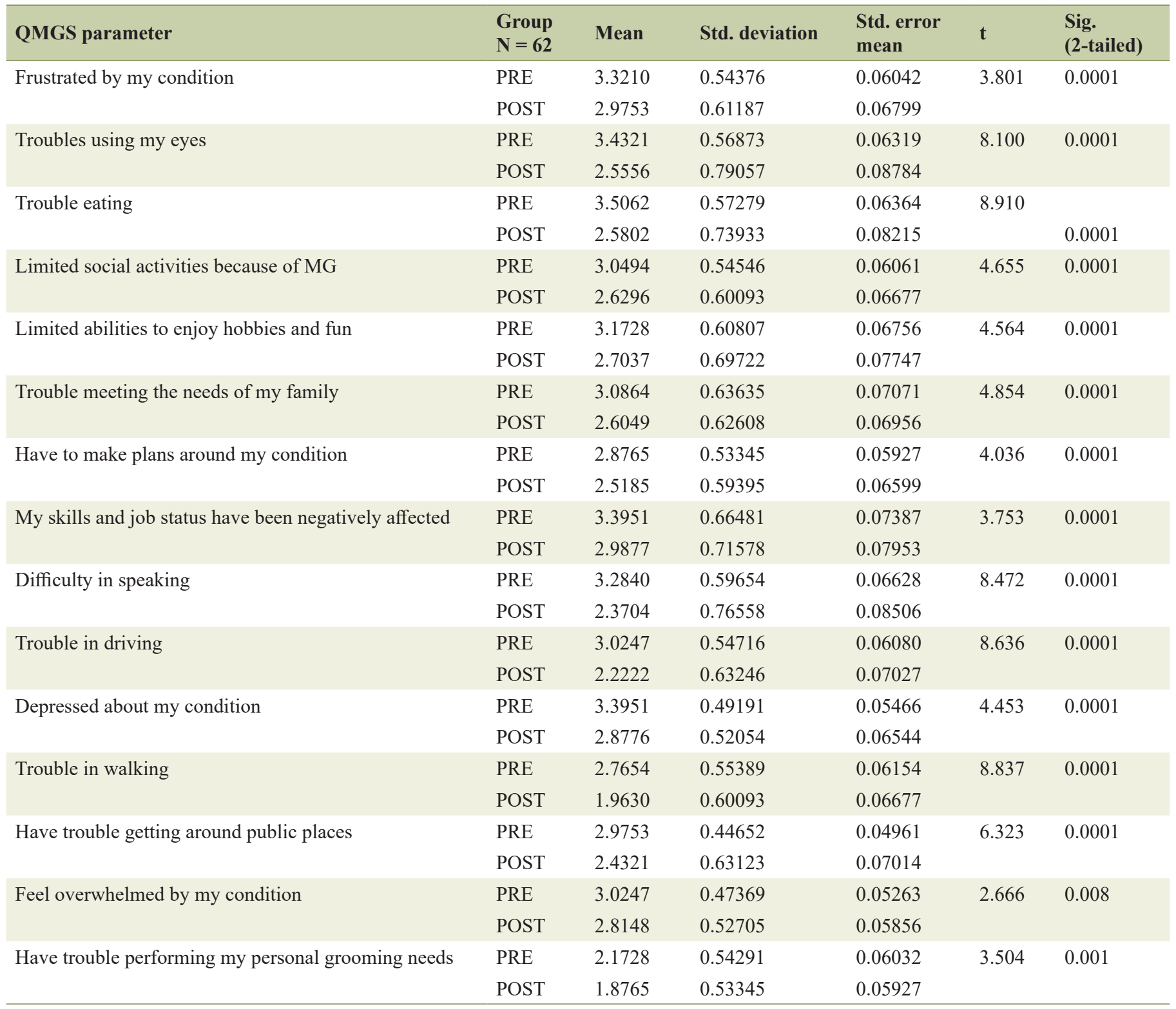

QMGS: Quantitative Myasthenia Gravis Scale; TPE: therapeutic plasma exchange; PRE: before TPE; POST: after TPE. P > 0.05: non-significance difference, $P<0.05$ : significant difference; $P<0.01$ : highly significant difference.

may be partially a function of the serum anti-AChR antibody levels, neither the presence nor the absolute concentration of these antibodies accurately predicts prognosis or therapeutic response [11].

Our study showed that in patients with moderate to severe MG, the treatment with TPE led to a significantly better response than the medical treatment only. Also, the response to TPE was significantly greater in sero-positive patients than in the sero-negative ones.

Gajdos et al in their meta-analysis on TPE in MG proved that TPE gives short term improvement in patients with MG especially in those with exacerbations or myasthenic crisis [12]. The advances in apheresis machines have made it a very safe modality of treatment and clinical improvement can be started within a day to a week [13]. Rapidly reducing the autoantibodies by TPE may lead to a rebound overproduction of the same antibodies making the replicating pathogenic cells more vulnerable to cytotoxic drugs. For this reason, it is often performed to enhance the effectiveness of cytotoxic drugs [14]. Our results were in agreement with Katzberg et al, who proved that AChR antibody positivity in addition to the baseline QMGS score was more abnormal in patients who responded to treatment. They had studied the factors that may predict the response to immunomodulation in patients with $\mathrm{MG}$ and proved that, the number of patients with AChR antibodies was greater in good responders, while sero-negative patients had a 

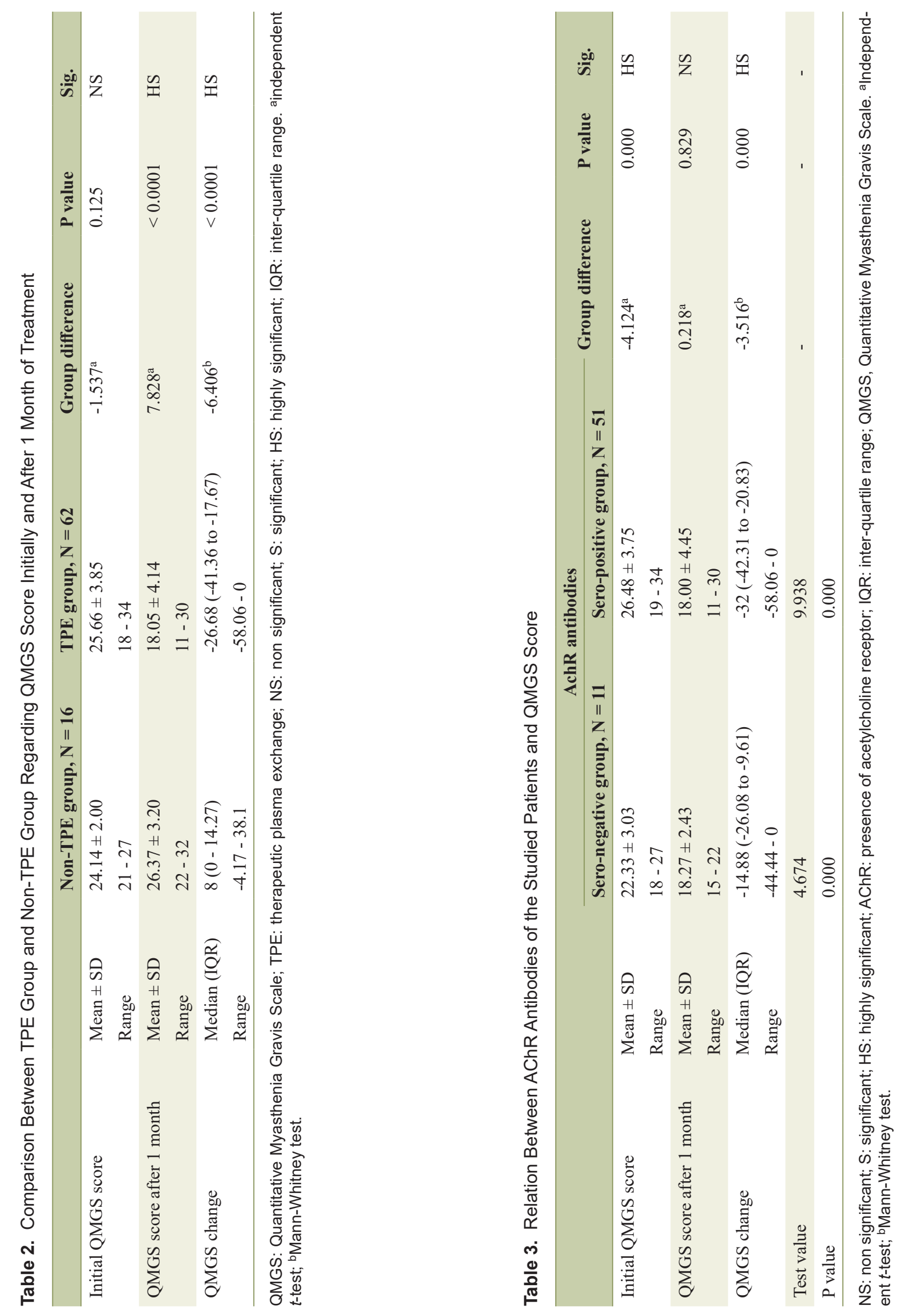
poor response [15].

On the other hand, many authors found that sero-positive MG patients had the same outcome as sero-negative patients, and serum $\mathrm{AChR} A b$ titers were not associated with MG prognoses [16-18]. Spillane et al in 2012 found that despite the higher proportion of responders in the sero-positive patients, some of sero-negative patients responded also to treatment, concluding that it is inappropriate to use AChR antibody status to determine treatment decisions [19]. Nils et al, during their study on the characteristics of MG autoantibodies, found that all MG subgroups, even the sero-negative one, responded with the same way to either intravenous immunoglobulin (IVIG) or TPE, suggesting that sero-negative patients may also have circulating pathogenic antibodies [20]. Rubin found in 2012 that, in comparison to the patients with MG who did not respond to TPE, the responders were more likely to be sero-positive for $\mathrm{AChR}$ antibodies, and tended to have higher baseline quantitative MG score and greater titter on single fiber electromyography. When using multivariate regression, only baseline QMGS proved to be the only significant predictor of response to TPE [21].

The binding of antibodies reduces the number and/or function of muscle AChR by the following three mechanisms: 1) Complement activation leading to destruction of the postsynaptic folds at the neuromuscular junction (NMJ) and destruction of AChR and AChR-related proteins at the motor end-plate; 2) Cross-linking of adjacent AChR causing their accelerated internalization and degradation; and 3) Blocking of the acetylcholine-binding area [22]. Although the exact mechanism of auto-sensitization to the AChR is not clear, abnormalities of the thymus gland (hyperplasia and neoplasia) may play a role in the majority of $M G$ patients. AChR-specific $\mathrm{CD} 4^{+} \mathrm{T}$ cells are present in the blood of the patients with these antibodies and are particularly copious in the thymus, supporting the concept that the thymus is the site in which the T-cell autosensitization occurs in MG [23].

One of the limitations of this study was that it studies the antibodies of AChR only. Further studies may be needed to evaluate if the presence of other antibodies of MG, such as the antibodies against muscle-specific tyrosine kinase (MuSK), may play a role in the prediction of the response to TPE.

\section{Conclusions}

As regards to the presence of $\mathrm{AChR}$ antibodies in patients with $\mathrm{MG}$, both sero-positive and sero-negative groups responded to TPE but the sero-positive group had a better response.

\section{Funding}

None.

\section{Conflict of Interest}

None.

\section{References}

1. McGrogan A, Sneddon S, de Vries CS. The incidence of myasthenia gravis: a systematic literature review. Neuroepidemiology. 2010;34(3):171-183.

2. Carr AS, Cardwell CR, McCarron PO, McConville J. A systematic review of population based epidemiological studies in Myasthenia Gravis. BMC Neurol. 2010;10:46.

3. Agius MA, Richman DP, Vincent A. Autoantibody testing in the diagnosis and management of autoimmune disorders of neuromuscular transmission and related diseases. In: Kaminski HJ, editor. Myasthenia Gravis and Related Disorders. 2. Humana Press; NJ, USA: 2009. pp. 143-156.

4. Masuda T, Motomura M, Utsugisawa K, Nagane Y, Nakata R, Tokuda M, Fukuda T, et al. Antibodies against the main immunogenic region of the acetylcholine receptor correlate with disease severity in myasthenia gravis. J Neurol Neurosurg Psychiatry. 2012;83(9):935-940.

5. Smith JW, Weinstein R, Hillyer KL, Committee AH, American Society for A. Therapeutic apheresis: a summary of current indication categories endorsed by the AABB and the American Society for Apheresis. Transfusion. 2003;43(6):820-822.

6. Deymeer F, Gungor-Tuncer O, Yilmaz V, Parman Y, Serdaroglu P, Ozdemir C, Vincent A, et al. Clinical comparison of anti-MuSK- vs anti-AChR-positive and seronegative myasthenia gravis. Neurology. 2007;68(8):609-611.

7. Robinson J, Eccher M, Bengier A, Liberman J. Costs and charges for plasma exchange (PLEX) versus intravenous immunoglobulin (IVIg) in the treatment of neuromuscular disease. Neurology. 2012;78:PD6.008.

8. Barnett C, Wilson G, Barth D, Katzberg HD, Bril V. Changes in quality of life scores with intravenous immunoglobulin or plasmapheresis in patients with myasthenia gravis. J Neurol Neurosurg Psychiatry. 2013;84(1):94-97.

9. Conti-Fine BM, Milani M, Kaminski HJ. Myasthenia gravis: past, present, and future. J Clin Invest. 2006;116(11):2843-2854

10. Romi F, Hong Y, Gilhus NE. Pathophysiology and immunological profile of myasthenia gravis and its subgroups. Curr Opin Immunol. 2017;49:9-13.

11. Liu JF, Wang WX, Xue J, Zhao CB, You HZ, Lu JH, Gu Y. Comparing the autoantibody levels and clinical efficacy of double filtration plasmapheresis, immunoadsorption, and intravenous immunoglobulin for the treatment of late-onset myasthenia gravis. Ther Apher Dial. 2010;14(2):153-160.

12. Gajdos P, Chevret S, Toyka K. Plasma exchange for myasthenia gravis. Cochrane Database Syst Rev. 2002;4:CD002275.

13. Kumar R, Birinder SP, Gupta S, Singh G, Kaur A. Therapeutic plasma exchange in the treatment of myasthenia gravis. Indian J Crit Care Med. 2015;19(1):9-13.

14. Heatwole C, Johnson N, Holloway R, Noyes K. Plasma exchange versus intravenous immunoglobulin for myasthenia gravis crisis: an acute hospital cost comparison study. J Clin Neuromuscul Dis. 2011;13(2):85-94.

15. Katzberg HD, Barnett C, Bril V. Predictors of response 
to immunomodulation in patients with myasthenia gravis. Muscle Nerve. 2012;45(5):648-652.

16. Mantegazza R, Baggi F, Antozzi C, Confalonieri P, Morandi $\mathrm{L}$, Bernasconi $\mathrm{P}$, Andreetta $\mathrm{F}$, et al. Myasthenia gravis (MG): epidemiological data and prognostic factors. Ann N Y Acad Sci. 2003;998:413-423.

17. Guillermo GR, Tellez-Zenteno JF, Weder-Cisneros N, Mimenza A, Estanol B, Remes-Troche JM, Cantu-Brito C. Response of thymectomy: clinical and pathological characteristics among seronegative and seropositive myasthenia gravis patients. Acta Neurol Scand. 2004;109(3):217221.

18. Romi F, Gilhus NE, Aarli JA. Myasthenia gravis: clinical, immunological, and therapeutic advances. Acta Neurol Scand. 2005;111(2):134-141.

19. Spillane J, Hayward M, Hirsch NP, Taylor C, Kullmann DM, Howard RS. Late recurrent thymoma in myasthe- nia gravis: a case series. J Neurol Neurosurg Psychiatry. 2012;83(10):1030-1031.

20. Nils Erik G, Geir Olve S, Fredrik R, Konstantinos L, Paraskevi Z, Socrates T. Myasthenia gravis - autoantibody characteristics and their implications for therapy. Nature Reviews Neurology. 2016;12:259-268.

21. Rubin M. Predicting response to IVIG or plasmapheresis in myasthenia gravis. Neurology alert. 2012;30(11):8492.

22. Meriggioli MN. Myasthenia gravis with anti-acetylcholine receptor antibodies. Front Neurol Neurosci. 2009;26:94-108.

23. Gomez AM, Van Den Broeck J, Vrolix K, Janssen SP, Lemmens MA, Van Der Esch E, Duimel H, et al. Antibody effector mechanisms in myasthenia gravis-pathogenesis at the neuromuscular junction. Autoimmunity. 2010;43(5-6):353-370. 